\title{
Emergent Surgical Embolectomy for Massive Pulmonary Embolism Causing Intraoperative Cardiac Arrest
}

\author{
Best Anyama, MD, MPH, ${ }^{1,2}$ Omar Viswanath, MD, ${ }^{3}$ Carolina De La Cuesta, MD, ${ }^{4}$ Murlikrishna Kannan, MD, ${ }^{5}$ \\ Michael Wittels, MD, ${ }^{6}$ Steve Xydas, MD, ${ }^{7}$ Alan David Kaye, MD, PhD, ${ }^{2}$ David A. Farcy, MD $^{4,8}$ \\ ${ }^{1}$ Department of Surgery, Mount Sinai Medical Center, Miami Beach, FL ${ }^{2}$ Department of Anesthesiology, Louisiana State University Health \\ Sciences Center, New Orleans, LA ${ }^{3}$ Department of Anesthesia, Critical Care, and Pain Medicine, Beth Israel Deaconess Medical Center, \\ Boston, MA ${ }^{4}$ Division of Critical Care, Mount Sinai Medical Center, Miami Beach, FL ${ }^{5}$ Integrated Anesthesia Medical Group, Manhattan \\ Beach, CA ${ }^{6}$ Division of Orthopedic Surgery, Mount Sinai Medical Center, Miami Beach, FL ${ }^{7}$ Department of Cardiac and Thoracic Surgery, \\ Mount Sinai Medical Center, Miami Beach, FL ${ }^{8}$ Department of Emergency Medicine, Mount Sinai Medical Center, Miami Beach, FL
}

Background: A massive pulmonary embolism (PE) is associated with high mortality once cardiac arrest occurs. Surgical embolectomy is indicated in patients who have massive $\mathrm{PE}$.

Case Report: A 62-year-old male emergently underwent surgical embolectomy after sustaining an intraoperative cardiac arrest caused by a massive PE during an open reduction with internal fixation of a closed comminuted midshaft tibia fracture. Postoperatively, the patient developed pulmonary hypertension and acute renal failure. He was treated with aerosolized epoprostenol for right ventricular strain secondary to pulmonary hypertension. He survived the hospital course and was discharged without any other major complications.

Conclusion: Surgical embolectomy is a viable option for massive PE, and aerosolized epoprostenol can be used as adjuvant treatment for right ventricular strain secondary to acute pulmonary hypertension.

Keywords: Embolectomy, heart arrest, hypertension-pulmonary, venous thrombosis

Address correspondence to David A. Farcy, MD, Chairman, Department of Emergency Medicine, Director of Emergency Department, Critical Care, Mount Sinai Medical Center, 4300 Alton Rd., Miami Beach, FL 33140. Tel: (305) 674-2948. Email: dfarcy@msmc.com

\section{INTRODUCTION}

A massive pulmonary embolism (PE) resulting in cardiac arrest is associated with a mortality rate up to $74 \% .{ }^{1}$ Massive PE refers to the sustained systemic hypotension or shock secondary to significant right ventricular failure. ${ }^{2}$ Surgical embolectomy is indicated in patients with massive PE. ${ }^{3}$ We present the case of a patient who emergently underwent open surgical embolectomy of the main pulmonary artery after intraoperative cardiac arrest from a massive PE during repair of a lower limb fracture. We also describe the use of aerosolized epoprostenol for adjuvant treatment of right ventricular strain secondary to acute pulmonary hypertension.

\section{CASE REPORT}

A 62-year-old male with a history of chronic obstructive pulmonary disease and smoking presented to the emergency department with a 3-day-old closed right midshaft tibia fracture. The patient had slipped and fallen and was initially evaluated at an outside institution. He was splinted with a dorsal splint that immobilized the ankle to above the knee; surgical fixation was recommended. The patient presented to our institution for evaluation and definitive management and was admitted for an open reduction with internal fixation scheduled for the following day.

In the operating room, after standard American Society of Anesthesiology monitors were placed, general anesthesia was induced with lidocaine, propofol, fentanyl, and succinylcholine, and the airway was secured via 8- $\mathrm{mm}$ endotracheal tube and Miller 3 blade. The patient's hemodynamic parameters were stable during the first 30 minutes: blood pressure (BP) of $135 / 85 \mathrm{mmHg}$, heart rate of $95 \mathrm{bpm}$ in normal sinus rhythm, and oxygen saturation of $100 \%$. A tourniquet was applied because of excessive bleeding. After rod insertion, the patient's heart rate increased to $115 \mathrm{bpm}$, oxygen saturation dropped from $100 \%$ to $70 \%$, end-tidal carbon dioxide $\left(\mathrm{CO}_{2}\right)$ dropped from $42 \mathrm{mmHg}$ to $24 \mathrm{mmHg}$, and arterial blood gas demonstrated severe metabolic respiratory acidosis and life-threatening hypoxia $\left(\mathrm{pH}\right.$ 6.97, $\mathrm{PCO}_{2}$ $97 \mathrm{mmHg}, \mathrm{PO}_{2} 48 \mathrm{mmHg}$ on an $\mathrm{FiO}_{2}$ of $100 \%$, base deficit $-6.3 \mathrm{mmol} / \mathrm{L}$ ) (Figure 1). Vasopressors were administered to mitigate the acute hypotension that ensued. Massive PE was suspected.

Because of the patient's cardiovascular deterioration, the orthopedic surgeon locked the rod proximally, quickly closed the wound with staples, and removed the tourniquet. Central venous access was obtained and an arterial line was 


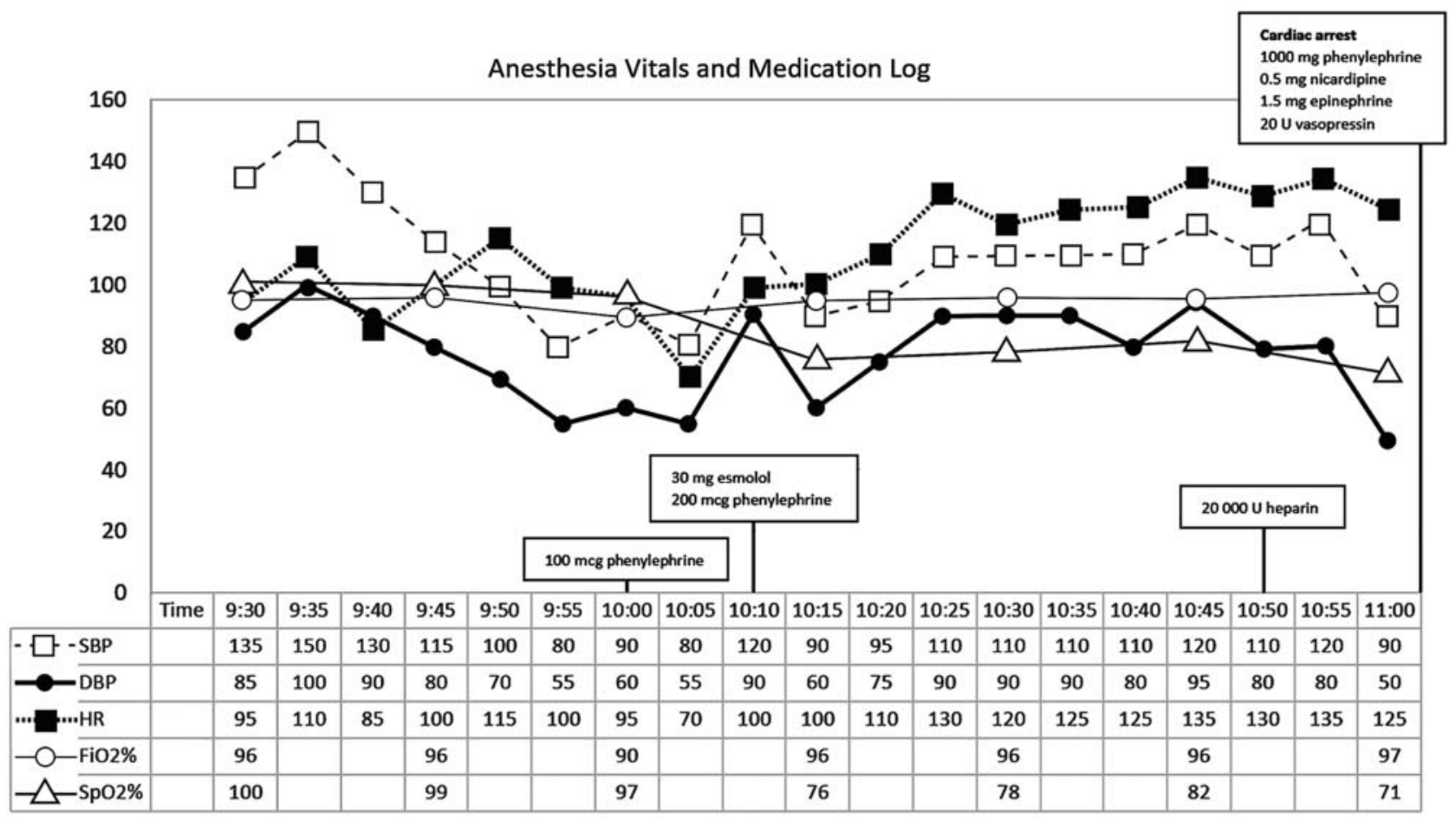

Figure 1. Log of the patient's vitals and drugs administered during the orthopedic procedure. A drop in systolic blood pressure and tachycardia occurred at 09:45 am. Severe hypotension occurred at 09:55 am, and phenylephrine and esmolol were administered. Transesophageal echocardiogram revealed right ventricular dysfunction and thrombus in the pulmonary artery and possible thrombus in the right atrium. Heparin was administered at 10:50 am. Ventricular fibrillation and the subsequent initiation of advanced cardiac life support occurred at 11:00 am.

placed. Intraoperative transesophageal echocardiography (TEE) demonstrated a large saddle PE (Figure 2) with fulminant right ventricular failure and possible right atrial thrombus. The patient was given 20,000 units of heparin but went into ventricular fibrillation 10 minutes later. Spontaneous circulation returned after 1 minute of cardiopulmonary resuscitation with advanced cardiac life support protocols.

A cardiothoracic surgeon and perfusion team were mobilized to the operating room where the patient was placed on cardiopulmonary bypass after sternotomy and central arterial and venous cannulation of the distal ascending aorta and superior and inferior vena cava. He was cooled to $32^{\circ} \mathrm{C}$. The cardiothoracic surgeon induced cardioplegia to prevent further ischemic damage to myocytes. After the aorta was cross-clamped, a longitudinal incision was made in the main pulmonary artery and a large embolus was removed (Figure 3). The distal pulmonary arteries were examined with a Fogarty balloon and found to be free of additional emboli. Thrombus was also removed from the right atrium and ventricle (Figure 3). After pulmonary arteriorrhaphy was completed, the aortic cross-clamp was released and an organized rhythm of the heart was restored after placement of a cardiac pacing wire. The patient came off cardiopulmonary bypass with improvement of right ventricular function. He was transported to the cardiac intensive care unit intubated, sedated, and in stable condition.

A heparin infusion for anticoagulation was maintained at a rate that achieved a partial thromboplastin time of 66-92 seconds, and hemodynamic support was achieved with norepinephrine at an infusion rate that maintained a mean arterial pressure goal of $59-70 \mathrm{mmHg}$. Aerosolized epoprostenol was started at $2 \mathrm{ng} / \mathrm{kg} / \mathrm{min}$ for pulmonary hypertension (pulmonary artery range of $30-40 \mathrm{mmHg}$ ) and was weaned during the course of 2 days postoperatively.

The patient's postoperative course was complicated by Haemophilus influenzae treated with ceftriaxone, ileus managed by gastric decompression, and acute renal failure that resolved after 15 days of continuous veno-venous hemodialysis. The patient was extubated on postoperative day (POD) 10. On POD 15, hemodialysis was discontinued, warfarin was initiated, and the patient was transferred to the floor. On POD 27, the patient was discharged home. When he was seen in the clinic 6 weeks postoperatively, he was at his baseline exercise tolerance. Formal locking of the distal rod by an orthopedic surgeon was to be scheduled.

\section{DISCUSSION}

PEs are categorized either as submassive or massive. Patients with a submassive PE have evidence of right ventricular dysfunction but are hemodynamically stable. Patients with a massive PE have severe right ventricular dysfunction, are not hemodynamically stable, and may have cardiogenic shock. $^{2}$

A massive PE resulting from a large thrombus can impede blood flow from the systemic to pulmonary vasculature, causing an acute increase in pulmonary vascular resistance that leads to pulmonary hypertension. The right ventricular myocytes, which are designed to work in a low-pressure system, are not able to sustain the mechanical strain and acute rise in metabolic demand. Cardiac output eventually 


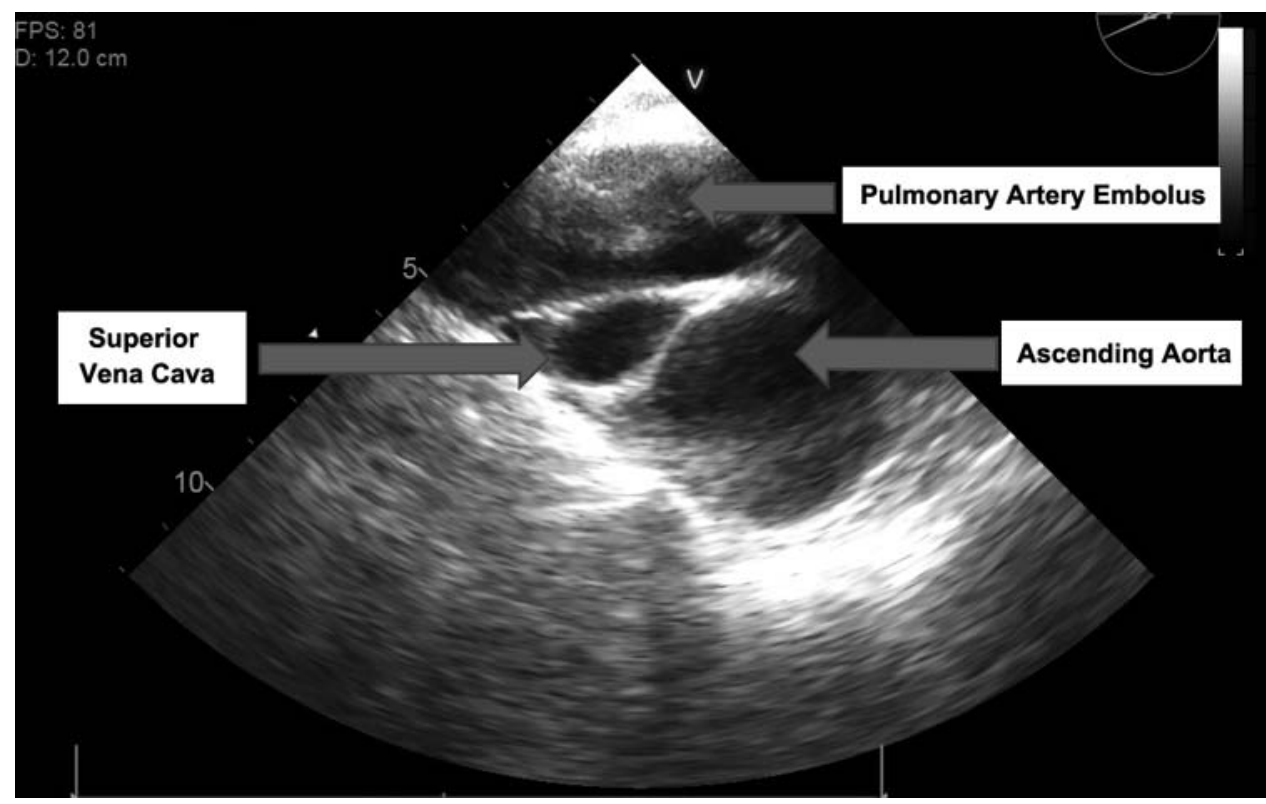

Figure 2. Transesophageal echocardiogram reveals a thrombus in the pulmonary artery.

decreases, and hemodynamic instability follows. ${ }^{4}$ A massive $\mathrm{PE}$ is associated with high mortality - up to $50 \%$ - within the first 6 hours, making the initial therapeutic management an important determinant in the patient's outcome..$^{5,6}$

Treatment selection depends on several factors, including the risk of bleeding, stability of the patient, resource availability, and thrombus burden. ${ }^{5}$ Thrombolysis is considered the first-line intervention for PE. A 2016 case report reported favorable outcomes with the use of intravenous thrombolytic therapy administered intraoperatively in a patient undergoing repair of an open left tibial fracture. ${ }^{7}$ Thrombolysis is a relative contraindication, however, in intraoperative patients and has been associated with an increased rate of major bleeding and intracranial hemorrhage compared to anticoagulation therapy alone. ${ }^{7,8}$ Catheter-directed embolectomy is an alternative to thrombolysis, especially for patients who have contraindications or who have failed thrombolysis therapy; however, complications include distal thrombus embolization, right ventricular injury, arrhythmia, pulmonary hemorrhage, pericardial tamponade, and femoral venous injury. ${ }^{9}$

Surgical embolectomy is indicated for patients who have a PE that causes hemodynamic instability, particularly for patients who have failed or have contraindications for thrombolytic therapy or for those with a patent foramen ovale or right heart thrombus. ${ }^{3}$ Thrombus from the pulmonary arteries and the right side of the heart can be rapidly removed using open surgical embolectomy. ${ }^{10}$ Preoperative imaging is integral to planning the appropriate surgical approach. ${ }^{11}$ Usually, computed tomography angiography is obtained as part of the preoperative workup to determine the thrombus location and extent. A TEE is obtained to evaluate for right ventricular strain and dysfunction and can also determine if a patent foramen ovale is present. ${ }^{11}$ Surgery is typically successful when the PE is centrally located - namely in

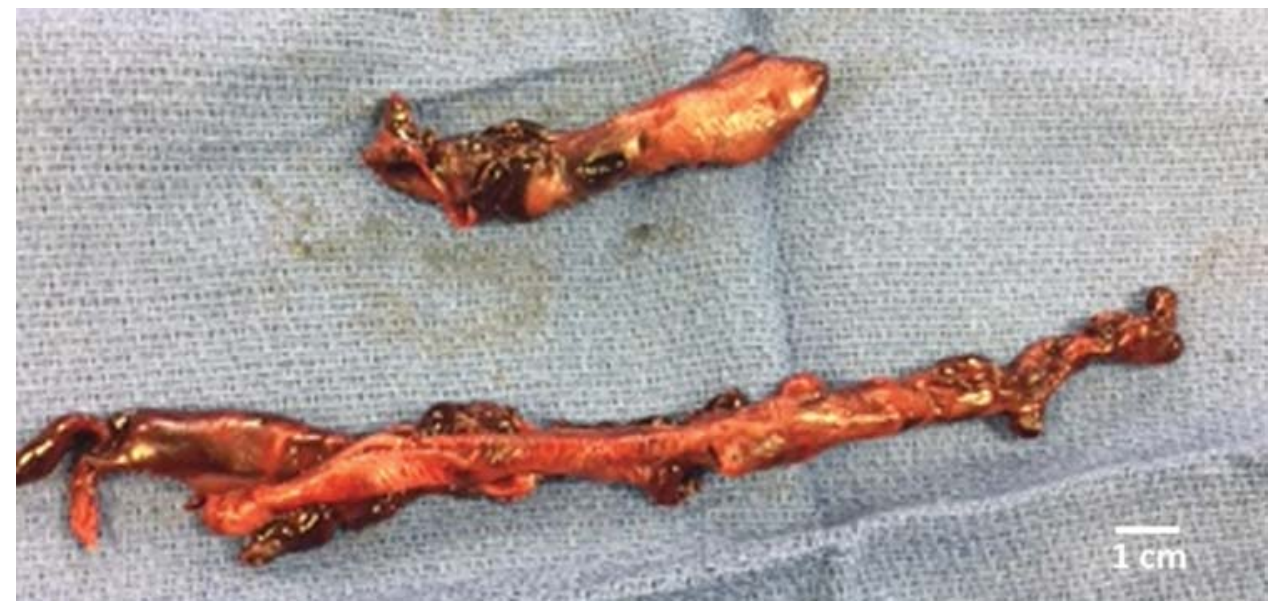

Figure 3. The thrombi extracted from the right atrium (above) and the pulmonary artery (below). 
the main, left, or right pulmonary arteries_and without extensive distal thrombus load. ${ }^{6}$

The overall mortality rate for massive $\mathrm{PE}$ has been reported to be $52.4 \%$. If a massive PE causes cardiac arrest, the mortality rate approaches $74 \%,{ }^{12,13}$ but the rate significantly decreases with surgical intervention. In a 2016 multiinstitutional study, the mortality rate for massive PE was $23.7 \%$ after surgical embolectomy. ${ }^{14}$ In our patient, TEE revealed saddle embolus in the pulmonary artery and a possible embolus in the right atrium. Consequently, both the pulmonary artery and the right atrium were opened to remove the thrombi.

Our patient sustained intraoperative cardiac arrest following an acute massive PE. He survived with surgical intervention and a 27-day postoperative hospital course. Rapid diagnosis and intervention, along with the patient's relatively preserved functional status prior to his hospitalization, increased his likelihood of survival. In healthy patients, hemodynamic instability and acute pulmonary hypertension secondary to right heart failure are reversible. ${ }^{15}$ Early and late death may also be reduced in patients without major comorbidities. ${ }^{4}$ However, once multiorgan dysfunction and cardiac arrest occur, the prognosis is poor. ${ }^{1,16}$

Patients may benefit from therapy selective for the pulmonary vasculature such as inhaled nitric oxide that improves ventilation-perfusion matching by increasing perfusion to areas of the lung that are well ventilated. Also, as a selective pulmonary vasodilator, inhaled nitric oxide has been suggested as a safe and effective agent to decrease the pulmonary vascular bed in patients with pulmonary hypertension and has been shown to have an effect similar to intravenous nitric oxide in pulmonary hypertension, although no mortality difference has been shown in long-term outcomes. ${ }^{17,18}$ In patients refractory to the above therapies, right ventricular assist device implantation should be considered. Our institution did not stock nitric oxide, but we used epoprostenol, a synthetic prostacyclin, as monotherapy to reduce the patient's pulmonary hypertension with the aim of reducing right ventricle strain caused by the massive PE. A 2017 randomized study found that prostacyclin significantly reduced pulmonary artery pressure and pulmonary vascular resistance and can be used as an alternative to nitric oxide for pulmonary hypertension therapy. ${ }^{19}$

From the time of fracture to his presentation at our hospital, the patient did not receive deep vein thrombosis (DVT) prophylaxis. He left the initial facility against medical advice because he wanted to receive care at our facility. In a case series of 115 patients undergoing surgical embolectomy, DVT was a risk factor for PE in $37 \%$ of patients. ${ }^{11}$ Although an ultrasound may have revealed DVT of the deep veins of the lower extremity prior to our patient's orthopedic surgery, this test was not performed. As a result, we have implemented a DVT ultrasound screening protocol to help prevent this kind of situation from recurring.

\section{CONCLUSION}

This case highlights the importance of clinicians being cognizant of acute decompensation secondary to massive PE; using proper tools such as TEE to confirm the diagnosis; proceeding immediately with surgical embolectomy to treat massive PE, even in cases of ongoing cardiac arrest; and considering aerosolized epoprostenol as a potential adjuvant treatment for right ventricular strain secondary to acute pulmonary hypertension in the intensive care unit. Contingent upon timely diagnosis and immediate intervention, surgical embolectomy for massive PE performed after cardiopulmonary collapse is feasible and can result in a favorable outcome.

\section{ACKNOWLEDGMENTS}

The authors have no financial or proprietary interest in the subject matter of this article.

\section{REFERENCES}

1. Kürkciyan I, Meron G, Sterz F, et al. Pulmonary embolism as a cause of cardiac arrest: presentation and outcome. Arch Intern Med. 2000 May 22;160(10):1529-1535.

2. Konstantinides SV, Barco S, Lankeit M, Meyer G. Management of pulmonary embolism: an update. J Am Coll Cardiol. 2016 Mar 1;67(8):976-990. doi: 10.1016/j.jacc.2015.11.061.

3. Samoukovic G, Malas T, deVarennes B. The role of pulmonary embolectomy in the treatment of acute pulmonary embolism: a literature review from 1968 to 2008. Interact Cardiovasc Thorac Surg. 2010 Sep;11(3):265-270. doi: 10.1510/ icvts.2009.228361.

4. Cho YH, Sung K, Kim WS, et al. Management of acute massive pulmonary embolism: is surgical embolectomy inferior to thrombolysis? Int J Cardiol. 2016 Jan 15;203:579-583. doi: 10.1016/j.ijcard.2015.10.223.

5. Cooper JM, Beckman JA. Massive pulmonary embolism: a remarkable case and review of treatment. Vasc Med. 2002 Aug;7(3):181-185.

6. Moorjani N, Price S. Massive pulmonary embolism. Cardiol Clin. 2013 Nov;31(4):503-518. doi: 10.1016/j.ccl.2013.07.005.

7. Kostetskiy IV, Agalakov MV, Tukhanov VV, Gracheva GV. Case of intersurgical acute massive pulmonary embolism with successful thrombolysis therapy. Oxf Med Case Reports. 2016 Aug;2016(8).

8. Chatterjee S, Chakraborty A, Weinberg I, et al. Thrombolysis for pulmonary embolism and risk of all-cause mortality, major bleeding, and intracranial hemorrhage: a meta-analysis. JAMA. 2014 Jun 18;311(23):2414-2421. doi: 10.1001/jama.2014.5990.

9. Lapidus LJ, Ponzer S, Pettersson H, de Bri E. Symptomatic venous thromboembolism and mortality in orthopaedic surgery-an observational study of 45968 consecutive procedures. BMC Musculoskelet Disord. 2013 Jun 4;14:177. doi: 10.1186/1471-2474-14-177.

10. Kabrhel C, Rempell JS, Avery LL, Dudzinski DM, Weinberg I. Case records of the Massachusetts General Hospital: case 292014: a 60-year-old woman with syncope. N Engl J Med. 2014 Sep 18;371(12):1143-1150. doi: 10.1056/NEJMcpc1403307.

11. Neely RC, Byrne JG, Gosev I, et al. Surgical embolectomy for acute massive and submassive pulmonary embolism in a series of 115 patients. Ann Thorac Surg. 2015 Oct;100(4):1245-1251. doi: 10.1016/j.athoracsur.2015.03.111.

12. Ahmed $P$, Khan AA, Smith A, et al. Expedient pulmonary embolectomy for acute pulmonary embolism: improved outcomes. Interact Cardiovasc Thorac Surg. 2008 Aug;7(4):591594. doi: 10.1510/icvts.2008.176735.

13. Kucher N, Rossi E, De Rosa M, Goldhaber SZ. Massive pulmonary embolism. Circulation. 2006 Jan 31;113(4):577-582.

14. Keeling WB, Sundt $T$, Leacche $M$, et al; SPEAR Working Group. Outcomes after surgical pulmonary embolectomy for acute pulmonary embolus: a multi-institutional study. Ann Thorac Surg. 2016 Nov;102(5):1498-1502. doi: 10.1016/j. athoracsur.2016.05.004. 
15. Chen HL, Wong CS, Ho ST, Chang FL, Hsu CH, Wu CT. A lethal pulmonary embolism during percutaneous vertebroplasty. Anesth Analg. 2002 Oct;95(4):1060-1062.

16. Bĕlohlávek J, Dytrych V, Linhart A. Pulmonary embolism, part II: management. Exp Clin Cardiol. 2013 Spring;18(2):139-147.

17. Ichinose F, Roberts JD Jr, Zapol WM. Inhaled nitric oxide: a selective pulmonary vasodilator: current uses and therapeutic potential. Circulation. 2004 Jun 29;109(25):3106-3111.
18. Kavanagh BP, Pearl RG. Inhaled nitric oxide in anesthesia and critical care medicine. Int Anesthesiol Clin. 1995 Winter;33 (1):181-210.

19. Abe $S$, Ishida $K$, Masuda $M$, et al. A prospective, randomized study of inhaled prostacyclin versus nitric oxide in patients with residual pulmonary hypertension after pulmonary endarterectomy. Gen Thorac Cardiovasc Surg. 2017 Mar;65(3): 153-159. doi: 10.1007/s11748-016-0724-2.

This article meets the Accreditation Council for Graduate Medical Education and the American Board of Medical Specialties Maintenance of Certification competencies for Patient Care and Medical Knowledge. 